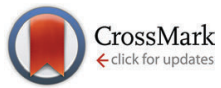

Cite this: J. Mater. Chem. C, 2015, 3, 9379

\title{
Programming the assembly of gold nanoparticles on graphene oxide sheets using DNA $\dagger$
}

\author{
Amelie Heuer-Jungemann, ${ }^{a}$ Liam Kiessling, ${ }^{a}$ Emmanuel Stratakis, ${ }^{\text {bc }}$ \\ Emmanuel Kymakis, ${ }^{d}$ Afaf $\mathrm{H}$. El-Sagheer, ${ }^{\text {ef }}$ Tom Brown $^{f}$ and Antonios G. Kanaras ${ }^{\star a g}$
}

\begin{abstract}
We present a new method to program the covalent binding of gold nanoparticles onto graphene oxide sheets. The binding selectivity is driven by the synergy of chemically modified oligonucleotides, grafted onto the surfaces of nanoparticles and graphene oxide. In the presence of a templating complementary DNA strand, nanoparticles are brought near the surface of the graphene oxide. Once in close proximity, the DNA strands are ligated to create a permanent link between the nanoparticles and graphene oxide, ensuring stability of the system even during DNA melting conditions. Due to the selectivity and specificity of DNA, a second layer of gold nanoparticles of different size can be grafted on the top of the first layer of particles. The simplicity of this new method allows for its universal applicability when the formation of highly programmable, covalently linked hybrid nanoparticle-graphene oxide structures is a

necessity.
\end{abstract}

Received 3rd July 2015,

Accepted 17th August 2015

DOI: $10.1039 /$ c5tc01999k

www.rsc.org/MaterialsC

\section{Introduction}

Ground-breaking work by Novoselov and Geim in $2004^{1}$ reported for the first time a new crystallographic two-dimensional form of carbon, namely graphene. Very soon, it was realized that graphene possesses intrinsic electronic, optical and thermal properties. ${ }^{2}$ These unique properties of graphene, in combination with high flexibility, strength and the ease of its chemical modification, resulted in an immense rise of scientific interest. ${ }^{2-5}$ Lately, a significant amount of research on graphene and its derivatives has been focussed on the development of hybrid systems, where graphene is functionalized with other materials (e.g. nanoparticles (NP), active molecules) with the aim of developing novel nanostructures with enriched properties. $^{6-11}$ The applications of such systems span a broad range of research fields ranging from biomedicine to energy

${ }^{a}$ Physics and Astronomy, Faculty of Physical Sciences and Engineering, University of Southampton, Southampton, SO17 1BJ, UK. E-mail: a.kanaras@soton.ac.uk

${ }^{b}$ Institute of Electronic Structure and Laser (IESL) Foundation for Research and Technology-Hellas (FORTH), Heraklion, 71110 Crete, Greece

${ }^{c}$ Department of Materials Science and Technology, University of Crete, Heraklion, 71003 Crete, Greece

${ }^{d}$ Center of Materials Technology and Photonics \& Electrical Engineering Department Technological Education Institute (TEI) of Crete, Heraklion, 71004 Crete, Greece

${ }^{e}$ Chemistry Branch, Department of Science and Mathematics, Faculty of Petroleum and Mining Engineering, Suez University, Suez 43721, Egypt

${ }^{f}$ Department of Chemistry, University of Oxford, Chemistry Research Laboratory, 12 Mansfield Road, Oxford, OX1 3TA, UK

${ }^{g}$ Institute for Life Science, University of Southampton, Southampton, SO17 1BJ, UK

$\dagger$ Electronic supplementary information (ESI) available. See DOI: 10.1039/c5tc01999k harvesting. For example Stratakis et al. reported that graphene oxide (GO)-gold hole transport layers can significantly enhance the efficiency and stability of photovoltaic devices. ${ }^{12}$ Zhang and co-workers showed that quaternary composites of graphene@$\mathrm{Fe}_{3} \mathrm{O}_{4} @ \mathrm{SiO}_{2} @$ @olyaniline possess great potential as novel lightweight microwave absorption materials. ${ }^{10}$ Recently, our group demonstrated a DNA sensor based on fluorescence resonance energy transfer (FRET) between NaYF4:Yb,Er nanoparticles and graphene oxide, ${ }^{13}$ whilst Kim and co-workers reported the use of reduced GO-magnetite hybrids for the efficient removal of arsenic from drinking water. ${ }^{14}$ Choe et al. demonstrated an improved electrocatalytic oxygen reduction reaction using reduced graphene oxide (rGO) modified with PEDOT and $\mathrm{MnO}_{2}$ nanoparticles. ${ }^{11}$ On the other hand, Gilbertson et al. highlighted that the formation of hybrid plasmonic metal-graphene systems is especially attractive due to combined unique optical properties of both materials, resulting in a new class of optical metamaterials. ${ }^{15}$ From all these recent studies, it is evident that graphene and graphene derivative hybrid systems display enormous potential for a variety of applications. Thus, the requirement for novel strategies to create more complex and advanced graphene oxide-NP hybrid structures in a controlled manner becomes apparent.

However, most chemical protocols for the formation of graphene oxide-NP hybrid assemblies reported to date lack the element of specificity and programmability, and in many cases these protocols describe the uncontrollable adsorption of NPs onto the surface of graphene. This chemical approach is advantageous because of its simplicity, but it imposes 
limitations with respect to the controlled development of hybrid structures. To address this issue we utilized DNA - an excellent tool to create programmable GO-nanoparticle hybrid structures. Apart from its unique versatility and selectivity, DNA can be easily modified with functional groups to enrich its properties such as fluorescent dyes and chemically reactive groups. ${ }^{16}$ Thus, DNA has become an established scaffold for the assembly of novel nanomaterials. ${ }^{17-20}$

Here we show that oligonucleotides can be employed to rationally program the binding of gold nanoparticles on graphene oxide templates. Such hybrid assemblies have shown great potential applications in areas such as biosensing, MEMS or catalysis. $^{8,21,22}$ Moreover, the utilization of chemically modified oligonucleotides enables the use of copper-free click chemistry for the facile formation of DNA ligated hybrid graphene oxide-nanoparticle structures that remain robust even under stringent or DNA denaturing conditions. To the best of our knowledge this is the first report of the formation of highly programmable, covalently linked GO-AuNP hybrids.

\section{Results and discussion}

\section{Covalent attachment of oligonucleotides on GO}

GO contains a large number of carboxylic groups, which could be readily reacted with amine terminated molecules using an
EDC/sulfo-NHS coupling strategy. Therefore, in order to covalently bind DNAs on the GO surface we modified oligonucleotides (Oligo 1 and Oligo 5, see Scheme 1 for sequences) with an amine group. Because in this particular experiment our aim was to maximize the number of DNA strands attached to the GO, we employed a previously reported coupling protocol developed by our group, which has proven efficient for nanoparticulate systems. ${ }^{23}$

To ensure that GO was covalently functionalized with oligonucleotides, the samples were characterized by UV-vis spectroscopy, $\zeta$-potential measurements and FT-IR (characterisation was carried out after purification by three centrifugation/decantation and re-dispersion steps to ensure that any non-covalently attached DNA was removed).

The characteristic UV spectrum of GO exhibits a maximum peak at $230 \mathrm{~nm}$, owing to $\pi \rightarrow \pi^{*}$ transitions ( $\mathrm{C}=\mathrm{C}$ bonds) as well as a shoulder from $290-350 \mathrm{~nm}$ attributed to $\mathrm{n} \rightarrow \pi^{*}$ transitions ( $\mathrm{C}=\mathrm{O}$ bonds). ${ }^{24}$ Once the DNA is covalently bound, it alters the electronic ground state of GO, resulting in spectral changes as shown in Fig. 1A. ${ }^{25}$ The main characteristics of the spectrum in this case are the appearance of an absorption maximum at $260 \mathrm{~nm}$, indicative of the $\pi \rightarrow \pi^{*}$ transitions in the DNA strands, and a decrease of the shoulder at $280-350 \mathrm{~nm}$ (GO $\mathrm{n} \rightarrow \pi^{*}$ transition). (As a comparison, the UV-vis spectrum of DNA non-covalently adsorbed onto GO is shown in Fig. S7, ESI $\dagger$ ).

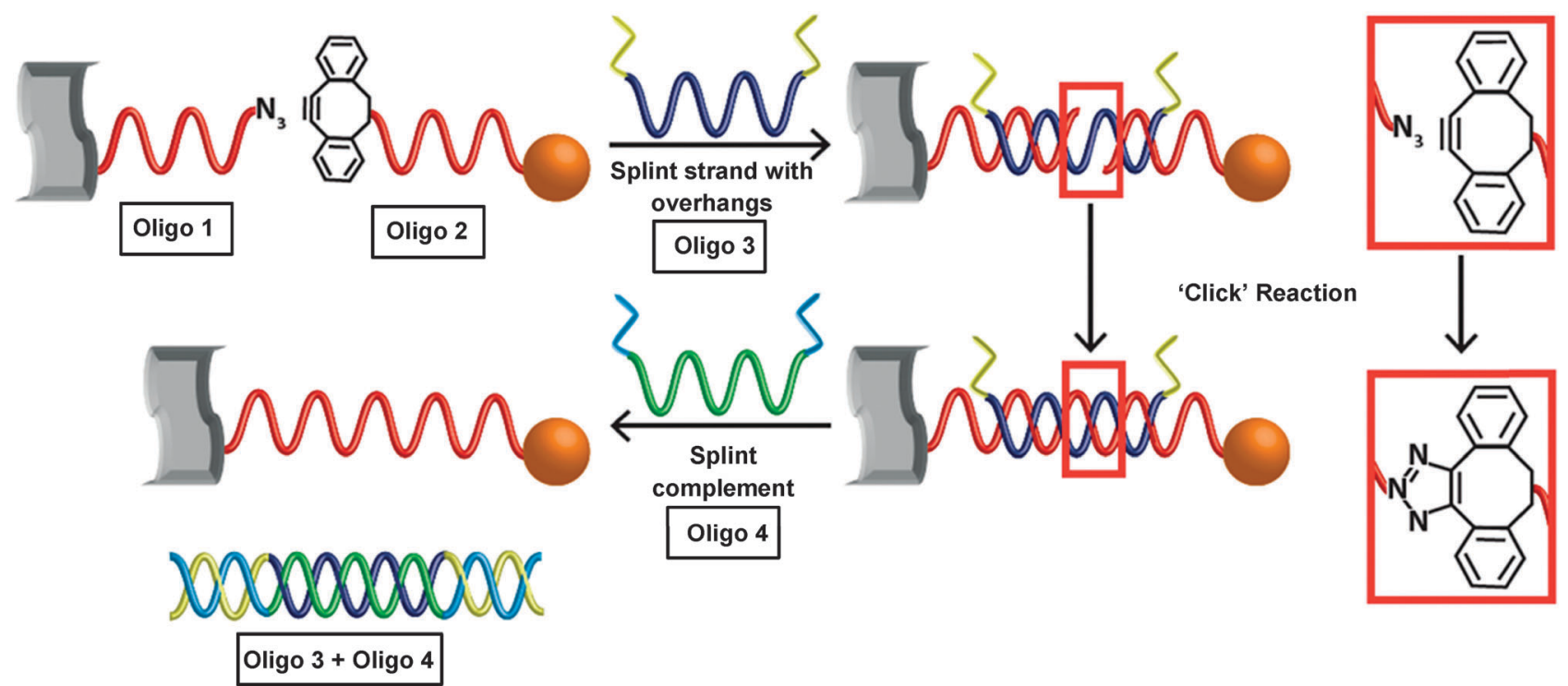

\begin{tabular}{|c|c|c|}
\hline Oligo 1 & 5'-(X)TTTTTTTTCGAGTGCTAAGGATCCGAAR, & \\
\hline Oligo 2 & 5'-R $\mathbf{R}_{2}$ TTCACTGCAGATATCCATTCGAAAAAAAA(X) & $R_{1}:$ Azide \\
\hline Oligo 3 & 5'-GTGTGTCGAGTGCTAAGGATCCGAATTCACTGCACATATCCATTCGGTGTGT & $R_{2}:$ Alkyne \\
\hline Oligo 4 & 5'-GTGTGTCGAGTGCTAAGGATCCGAATTCACTGCAGATATCCATTCGGTGTGT & $\mathrm{X}: \mathrm{C}_{6} \mathrm{H}_{12} \mathrm{NH}_{2} \mathrm{OR} \mathrm{C}_{6} \mathrm{H}_{12} \mathrm{SH}$ \\
\hline Oligo 5 & 5'-(X)TTTTTTTTCGAGTGCTAAGGATCCGAA & \\
\hline
\end{tabular}

Scheme 1 Schematic illustration of the formation of covalently ligated hybrid GO-AuNP systems. Oligo 1 and Oligo 2 DNA modified graphene oxide and nanoparticles are brought into close proximity by hybridisation with the templating splint strand Oligo 3. Once hybridised, click ligation occurs instantaneously, yielding a covalent triazole link. (For simplicity only one DNA strand per nanoparticle or graphene oxide sheet is shown). Owing to Oligo 3 bearing non-complementary overhangs (depicted in yellow), removal is possible by competitive hybridisation with the splint complement Oligo 4 (depicted in blue and green). 


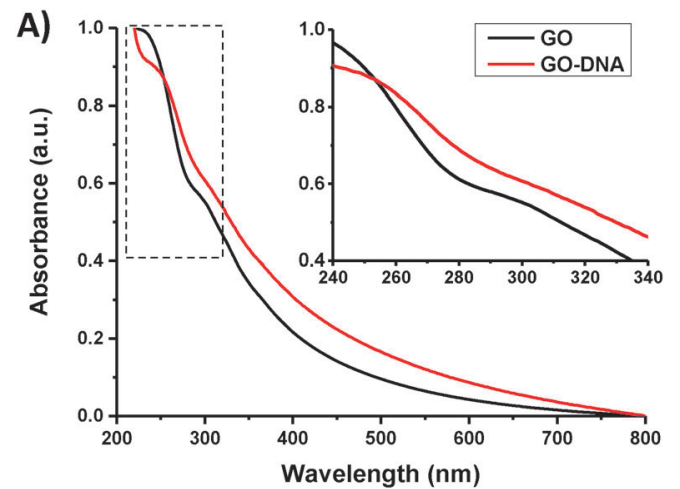

B)

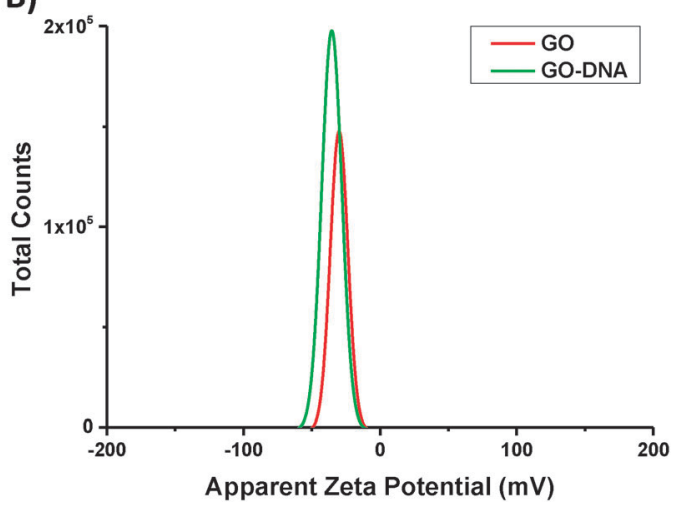

Fig. 1 (A) Normalized UV-vis spectra of GO (black) and DNA-modified $\mathrm{GO}$ (red). Inset: Magnification of spectral region in the boxed area. (B) $\zeta$-potential graphs of GO (red) and GO-DNA (green).

The functionalization of $\mathrm{GO}$ with oligonucleotides was also confirmed by $\zeta$-potential measurements, which showed a change in the net charge of the solution from $-25.2 \pm 0.4 \mathrm{mV}$ to $-40.1 \pm 1.4 \mathrm{mV}$ (Fig. 1B). This can be attributed to the increased negative charge conferred by the anionic DNA phosphate backbone.

Additional confirmation of covalent bonding between DNA and GO was obtained by FT-IR spectroscopy, which shows the appearance of characteristic amide absorption bands (Fig. S8, ESI $\dagger$ ).
The covalent functionalization of GO with DNA is essential to ensure that GO-DNA conjugates remain functional for further modifications even under conditions, which cause extensive DNA desorption such as low salt concentration or high $\mathrm{pH}^{26}$

\section{Formation of graphene oxide-gold nanoparticle hybrid assemblies}

The general strategy for the formation of graphene oxide-gold nanoparticle hybrid assemblies is illustrated in Scheme 1. In our experiment, an excess of alkyne-oligonucleotide (Oligo 2) coated $13 \mathrm{~nm}$ gold nanoparticles (see ESI, $\dagger$ Section S1 for the experimental preparation of DNA coated gold nanoparticles and Section S3 for the relevant characterization) was mixed with a solution of azide-oligonucleotide (Oligo 1) functionalized GO. Then a third template DNA strand (Oligo 3), partially complementary to both Oligo 1 and Oligo 2, was added to the solution. Once hybridized, the reactive alkyne and azide groups on Oligo 1 and Oligo 2 are situated in close proximity and spontaneously 'click'. Using a strained cyclooctyne as the reactive alkyne group allows for a spontaneous, copper-free click reaction with an azide group via the ring-strain promoted alkyne-azide [3+2] cycloaddition (SPAAC). ${ }^{27}$ Optionally, it is possible to remove Oligo 3 with the splint complement Oligo 4 via competitive hybridisation, due to the incorporation of non-complementary overhangs in Oligo 3 (see Scheme 1, overhangs are depicted in yellow). This simple, high yielding copper-free click chemistry reaction has previously been employed by us to successfully form gold nanoparticle dimers and trimers. ${ }^{16}$ The selectivity of the proposed method enables a multi-step programming of the desired materials as presented here.

To purify the covalently linked GO-nanoparticle assemblies from non-reacted DNA-coated gold nanoparticles, we took advantage of their large size difference. Agarose gel electrophoresis is widely employed to separate particles according to their size and charge. This effective purification method has been reported by others to separate functionalized graphene oxide from small species. ${ }^{15,21,25,28,29}$ An agarose gel of our experiment is shown in Fig. 2A. Two distinct bands can be seen in this gel. A red band, corresponding to unconjugated
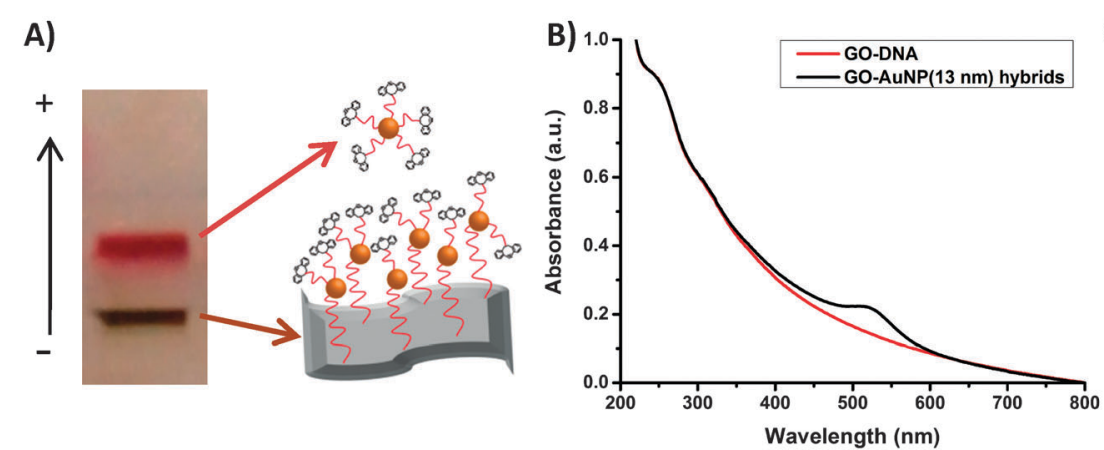

C)

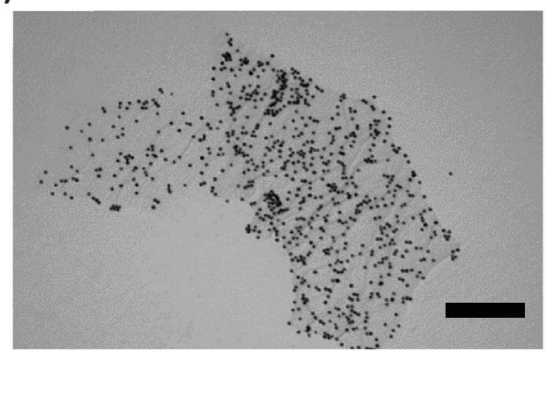

Fig. 2 (A) Purification of non-conjugated AuNPs (red band) from GO-AuNP hybrids (brown band); (B) normalized UV-vis spectra of GO-DNA (black) and GO-AuNP hybrids (red); (C) representative TEM image of GO-AuNP hybrids after purification. Scale bar is $500 \mathrm{~nm}$. 
DNA-AuNPs and a brown band corresponding to GO-gold nanoparticle conjugates. The small pore size of the agarose gel matrix allows only the comparatively small non-conjugated DNA-AuNPs to enter the gel, while the larger GO-AuNP assemblies remain in the wells. This accumulation in the gel allowed for facile separation of the pure hybrid nanoparticle-GO assemblies. Various techniques were utilized to show that AuNPs were successfully conjugated to GO.

Owing to their optical properties, UV-vis spectroscopy represents a quick and simple tool for the characterisation of hybrid assemblies. Fig. 2B displays the UV-vis spectra of GO modified with DNA (black) and pure GO-AuNP hybrids (red). As expected, the spectrum of the hybrid assemblies shows the appearance of the distinct gold nanoparticle plasmon peak at $520 \mathrm{~nm}$, indicating the presence of gold nanoparticles. ${ }^{25}$

Further evidence that the nanoparticles were successfully grafted onto GO was obtained by transmission electron microscopy. Fig. 2C shows a sheet of GO decorated with AuNPs (additional images and Raman spectra in ESI, $\dagger$ Fig. S9 and S10).

In order to confirm that AuNPs and GO sheets were indeed covalently ligated, a control experiment was performed (see $\mathrm{ESI}, \dagger$ Scheme S1). In this case assemblies were formed as before, but with GO-DNA conjugates lacking the azide functional group (Oligo 5-GO conjugates) required for the DNA click reaction. Following this experimental route, assemblies could still form in the presence of Oligo 3. However, as Oligo 3 was designed to contain non-complementary overhangs, it could be selectively removed, thus releasing the non-ligated DNA-nanoparticle conjugates from the graphene oxide template.

Fig. 3 displays a representative gel of ligated and non-ligated assemblies after removal of the templating splint strand Oligo 3 ( $c f$. Scheme 1 and Scheme S1, ESI $\dagger$ ). Lane 2 shows the clicked assemblies, (that had previously been purified as discussed above (cf. Fig. 2A)) as a brown-reddish band, which did not enter the agarose gel due to their large size. Contrastingly, in lane 1, a brown band could be seen in the well of the gel, accompanied by an additional red band. Due to the lack of an azide group on the Oligo 5-GO conjugates, the assemblies dissociated after removal of the splint strand, thus allowing the DNA-AuNP conjugates to enter the gel (red band), whilst GO-DNA conjugates remained in the well.
Subsequent analysis of the GO containing products from lanes 1 and 2 by UV-vis spectroscopy and TEM further confirmed these findings (see ESI, $\dagger$ Fig. S6). Whilst the TEM images show 'bare' GO sheets or assembled GO-AuNP hybrids for products recovered from lanes 1 and 2 respectively (Fig. S6B and $\mathrm{C}, \mathrm{ESI} \dagger$ ), UV-vis analysis (Fig. S6A, ESI $\dagger$ ) further revealed the disappearance of the gold nanoparticle plasmon peak in the spectrum of non-ligated assemblies. From these results it can be concluded that covalent functionalization of GO with AuNPs was indeed achieved successfully.

\section{Further functionalization of GO-AuNP hybrid assemblies}

In order to demonstrate that the formation of more complex hybrid assemblies can be programmed using our universal strategy, we performed a second click ligation step utilizing smaller, $5 \mathrm{~nm}$, AuNPs. These were functionalized with thiolated Oligo 1, thus being able to form assemblies only with Oligo 2-AuNPs grafted on the GO surface (see ESI, $\dagger$ Scheme S2).

As prepared GO-AuNP hybrids with attached DNA-AuNPs bearing free alkyne groups were mixed with an excess of $5 \mathrm{~nm}$ AuNPs, each of them bearing only one azide modified DNA strand (see ESI, $\dagger$ Fig. S5). These particles were then brought into close proximity via Oligo 3, allowing for the click ligation to occur. Subsequent purification by agarose gel electrophoresis finally yielded the pure hybrid structures of GO-AuNP (13 nm)AuNP (5 nm). The success of this additional grafting step was evident by UV-vis spectroscopy (Fig. 4A) and transmission electron microscopy (Fig. 4B).

As expected, the UV spectrum for GO-AuNP (13 nm)-AuNP (5 $\mathrm{nm}$ ) hybrid assemblies shows similarities to that of GO-AuNP (13 nm) assemblies. However, a broadening in the absorbance of the characteristic AuNP plasmon peak can be observed, indicating the successful ligation of $5 \mathrm{~nm}$ AuNP-DNA conjugates. Ultimately, transmission electron microscopy validated these results. Fig. 4B shows a GO sheet decorated with both 13 and $5 \mathrm{~nm}$ AuNPs (additional images in ESI, $\dagger$ Fig. S10). One can see that $5 \mathrm{~nm}$ AuNPs are indeed associated with $13 \mathrm{~nm}$ AuNPs, owing to their covalent DNA link.
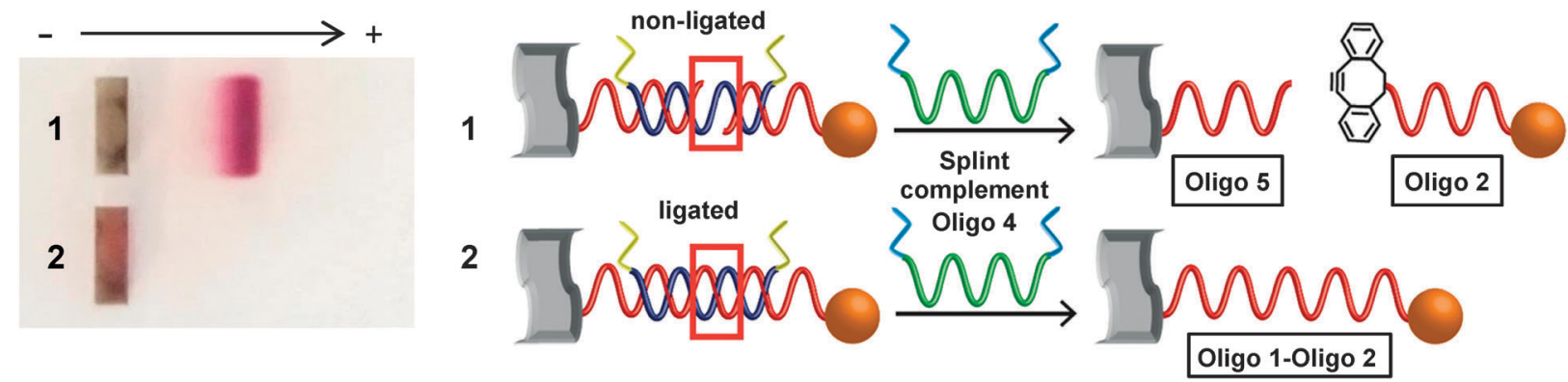

Fig. 3 A schematic illustration and a representative agarose gel of non-ligated (lane 1) and ligated (lane 2) hybrid assemblies. In both samples the templating splint strand Oligo 3 was removed using the splint complement strand Oligo 4. Assemblies that were previously ligated and purified remain intact (lane 2), whereas non-ligated assemblies dissociate (lane 1). 
A)

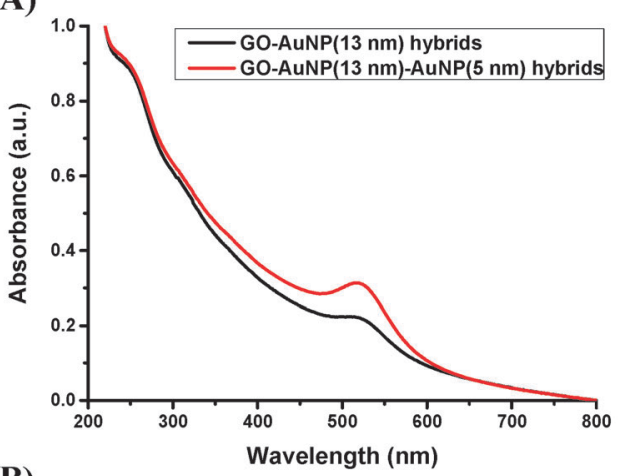

B)

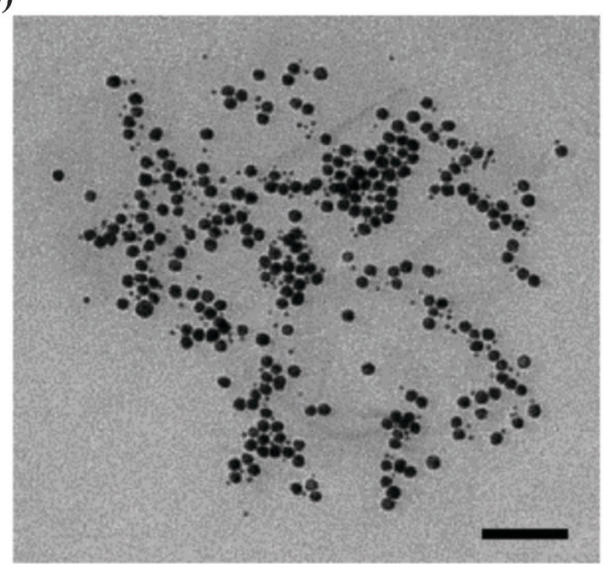

Fig. 4 (A) Normalized UV-vis spectra of GO-AuNP (13 nm) (black) and GO-AuNP (13 nm)-AuNP (5 nm) (red) hybrid assemblies; (B) TEM micrograph of GO-AuNP (13 nm)-AuNP ( $5 \mathrm{~nm}$ ) hybrid assemblies. Scale bar is $100 \mathrm{~nm}$.

\section{Conclusions}

Graphene/nanoparticle hybrids have seen a great rise in interest in the past years, yet there is a lack of specificity and programmability in many reported assembly strategies. In this study we showed that graphene oxide sheets can be decorated with $13 \mathrm{~nm}$ gold nanoparticles using copper free DNA click ligation. The programmability and specificity of our assembly strategy was successfully demonstrated by incorporation of a second layer of $5 \mathrm{~nm}$ gold nanoparticle-DNA conjugates. The described protocol represents a new and straightforward chemical route to create programmable and robust GO-nanoparticle hybrid structures. Due to the simple nature of the method, it will be universally applicable for the formation of even more complex advanced hybrid systems, unraveling a new era of functional materials.

\section{Acknowledgements}

The authors would like to thank ATDBio for synthesis of oligonucleotides and the Biomedical Imaging Unit, Southampton General Hospital. The financial support of the EPSRC program grant EP/G060363/1, the EPSRC Institutional Sponsorship grant (EP/K503575/1), the BBSRC: sLOLA grant BB/J001694/1 "Extending the boundaries of nucleic acid chemistry" and DSTL are gratefully acknowledged. AGK would also like to thank the EU COST action MP1202 for networking opportunities associated with this work.

\section{References}

1 K. S. Novoselov, A. K. Geim, S. V. Morozov, D. Jiang, Y. Zhang, S. V. Dubonos, I. V. Grigorieva and A. A. Firsov, Science, 2004, 306, 666-669.

2 A. K. Geim and K. S. Novoselov, Nat. Mater., 2007, 6, 183-191.

3 D. Chen, H. Feng and J. Li, Chem. Rev., 2012, 112, 6027-6053.

4 S. Roy, N. Soin, R. Bajpai, D. S. Misra, J. A. McLaughlin and S. S. Roy, J. Mater. Chem., 2011, 21, 14725-14731.

5 Y. Zhu, S. Murali, W. Cai, X. Li, J. W. Suk, J. R. Potts and R. S. Ruoff, Adv. Mater., 2010, 22, 3906-3924.

6 Y. Wang, S. Zhang, D. Du, Y. Shao, Z. Li, J. Wang, M. H. Engelhard, J. Li and Y. Lin, J. Mater. Chem., 2011, 21, 5319-5325.

7 X.-J. Lv, W.-F. Fu, H.-X. Chang, H. Zhang, J.-S. Cheng, G.-J. Zhang, Y. Song, C.-Y. Hu and J.-H. Li, J. Mater. Chem., 2012, 22, 1539-1546.

8 C. Li, Y. Yang, B. Zhang, G. Chen, Z. Wang and G. Li, Part. Part. Syst. Charact., 2014, 31, 201-208.

9 Z. S. Qian, X. Y. Shan, L. J. Chai, J. R. Chen and H. Feng, Biosens. Bioelectron., 2015, 68, 225-231.

10 L. Wang, J. Zhu, H. Yang, F. Wang, Y. Qin, T. Zhao and P. Zhang, J. Alloys Compd., 2015, 634, 232-238.

11 J. E. Choe, J. M. You, M. Yun, K. Lee, M. S. Ahmed, Z. Ustundag and S. Jeon, J. Nanosci. Nanotechnol., 2015, 15, 5684-5690.

12 E. Stratakis, M. M. Stylianakis, E. Koudoumas and E. Kymakis, Nanoscale, 2013, 5, 4144-4150.

13 P. Alonso-Cristobal, P. Vilela, A. El-Sagheer, E. Lopez-Cabarcos, T. Brown, O. L. Muskens, J. Rubio-Retama and A. G. Kanaras, ACS Appl. Mater. Interfaces, 2015, 7, 12422-12429.

14 V. Chandra, J. Park, Y. Chun, J. W. Lee, I. C. Hwang and K. S. Kim, ACS Nano, 2010, 4, 3979-3986.

15 A. M. Gilbertson, Y. Francescato, T. Roschuk, V. Shautsova, Y. Chen, T. P. H. Sidiropoulos, M. Hong, V. Giannini, S. A. Maier, L. F. Cohen and R. F. Oulton, Nano Lett., 2015, 15, 3458-3464.

16 A. Heuer-Jungemann, R. Kirkwood, A. H. El-Sagheer, T. Brown and A. G. Kanaras, Nanoscale, 2013, 5, 7209-7212.

17 D. Nykypanchuk, M. M. Maye, D. van der Lelie and O. Gang, Nature, 2008, 451, 549-552.

18 R. J. Macfarlane, B. Lee, M. R. Jones, N. Harris, G. C. Schatz and C. A. Mirkin, Science, 2011, 334, 204-208.

19 S. A. Claridge, A. J. Mastroianni, Y. B. Au, H. W. Liang, C. M. Micheel, J. M. J. Frechet and A. P. Alivisatos, J. Am. Chem. Soc., 2008, 130, 9598-9605.

20 C. J. Loweth, W. B. Caldwell, X. G. Peng, A. P. Alivisatos and P. G. Schultz, Angew. Chem., Int. Ed., 1999, 38, 1808-1812.

21 Z. H. Wang, Z. L. Ge, X. X. Zheng, N. Chen, C. Peng, C. H. Fan and Q. Huang, Nanoscale, 2012, 4, 394-399. 
22 J. Thavanathan, N. M. Huang and K. L. Thong, Biosens. Bioelectron., 2014, 55, 91-98.

23 D. Bartczak and A. G. Kanaras, Langmuir, 2011, 27, 10119-10123.

24 Q. Yang, X. Pan, K. Clarke and K. Li, Ind. Eng. Chem. Res., 2011, 51, 310-317.

25 F. Liu, J. Y. Choi and T. S. Seo, Biosens. Bioelectron., 2010, 25, 2361-2365.
26 J. Liu, Phys. Chem. Chem. Phys., 2012, 14, 10485-10496.

27 M. Shelbourne, X. Chen, T. Brown and A. H. El-Sagheer, Chem. Commun., 2011, 47, 6257-6259.

28 F. L. Bei, X. L. Hou, S. L. Y. Chang, G. P. Simon and D. Li, Chem. - Eur. J., 2011, 17, 5958-5964.

29 J. Liu, Y. Li, Y. Li, J. Li and Z. Deng, J. Mater. Chem., 2010, 20, 900-906. 